\title{
Implementation of three functional devices using erbium-doped fibers: an advanced photonics lab
}

\section{Wen Zhu, Li Qian, Amr Helmy}

Wen Zhu, Li Qian, Amr S. Helmy, "Implementation of three functional devices using erbium-doped fibers: an advanced photonics lab," Proc. SPIE 9665, Tenth International Topical Meeting on Education and Training in Optics and Photonics, 966511 (3 June 2007); doi: 10.1117/12.2207348

SDIE Event: Tenth International Topical Meeting on Education and Training in Optics and Photonics, 2007, Ottawa, Ontario, Canada 


\title{
Implementation of three functional devices using Erbium- doped Fibers: An Advanced Photonics Lab
}

\author{
Wen Zhu, Li Qian, Amr S. Helmy \\ Department of Electrical and Computer Engineering, University of Toronto, 10 King's College Road, Toronto, ON M5S 3G4, Canada \\ Tel: (416) 946-873, wen.zhu@utoronto.ca
}

\begin{abstract}
This paper describes the design and implementation of an advanced photonics experiment aimed at the undergraduate students' level. The experiment uses erbium-doped fiber to implement three functions through slight modifications of the setup. The functions are a broadband light source, a multi-wavelength optical amplifier, and a tunable fiber laser. As part of an Optical Communication Systems course, the experiment is targeted towards fourth year engineering students at the University of Toronto. The design of the experiment is especially attractive for large classes, where feasibility and cost effectiveness play a pivotal role. In addition the scope of the experiment was designed to illustrate a broad set of topics covered in the course, where students gain knowledge in: i) constructing a broadband source using the erbium-doped fiber amplified spontaneous emission (ASE) and characterize its emission spectrum; ii) modifying the ASE source into a broadband multi-wavelength erbium doped fiber amplifier (EDFA); iii) studying gain tilt and noise figure (NF) of the EDFA with respect to input and pump parameters; and finally, iv) transforming the EDFA into a tunable erbium doped fiber laser (EDFL). Through this series of experiments, students will (i) appreciate the versatility of an important optical gain medium; (ii) develop a deeper understanding of the salient features of optical gain including stimulated and spontaneous emission, principles of laser and amplifier action; (iii) learn, through hands on experience, to operate advanced optical components and test and measurement instruments which all form an integral part of the optical communication industry; and finally(iv) integrate the building blocks they have encountered in textbooks into operational optical devices.
\end{abstract}

\section{Introduction}

Since its invention in the late 1980s, the erbium-doped fiber has proved to be a versatile material system with a wide range of applications, including broadband optical sources, wide-band optical amplifiers, and tunable lasers. Broadband optical sources have been applied in various areas such as optical device characterization, gyroscopes, and optical coherence tomography. Amplified spontaneous emission (ASE) in erbium-doped fiber has been used to construct light sources with the advantages of high output power and broad optical bandwidth [1]. The erbiumdoped fiber amplifier (EDFA) was the first successful optical amplifier and has revolutionized the optical communication industry in the early 1990s. Today, they are widely used in all kinds of fiber communication systems, especially wavelength division multiplexed (WDM) systems [2] [3]. Erbium-doped fiber lasers (EDFL), one of the most popular fiber lasers, have shown tremendous progress in recent years. They have the advantages of good beam quality, wide tunable wavelength, small size, and lower cost etc. [4].

Lasers, optical amplifiers, especially EDFA, are all pivotal components in the state-of-the-art course of optical communication systems. The basic theory of optical amplifier and laser is a challenge for undergraduate students to grasp. Therefore it is essential to develop a practical experiment to help the students understand the basic performance of the optical amplifier and laser. Although there are EDFA education kits available in the market [5], we developed an experiment that use erbium-doped fiber to implement three functions: a broadband incoherent light source, a multi-wavelength optical amplifier, and a tunable fiber laser with more illustrative, flexible, and costive for large scale undergraduate course. The aim of the experiment is enable senior engineering students to: (i) construct an ASE broadband incoherent light source, an EDFA, and an EDFL based on same erbium-doped fiber and other components; (ii) characterize EDFA performance; (iii) establish deep understanding of the concepts of lasers, optical amplifiers; (iii) obtain practical experience of operating advanced optical components and instruments.

\section{Principles}

An erbium-doped fiber is an optical fiber of which the core is doped with rare-earth element erbium ions $\mathrm{Er}^{3+}$. A simplified energy level diagram of $\mathrm{Er}^{3+}$ ion is shown in Figure 1. The energy levels are broadening due to the dcStark effect [6], which leads to a relatively broad emission bandwidth. When a $974 \mathrm{~nm}$ pump laser diode beam is fed into an erbium-doped fiber, $\mathrm{Er}^{3+}$ will be excited from the ground state $\mathrm{E}_{1}$ to the higher level $\mathrm{E}_{3}$. The excited $\mathrm{Er}^{3+}$ ions on $E_{3}$ will rapidly decay to energy level $E_{2}$ through nonradiative emission. The excited ions on $E_{2}$ eventually return 
to ground state $\mathrm{E}_{1}$ through spontaneous emission, which produces photons in the wavelength band 1520 - $1570 \mathrm{~nm}$. The spontaneous emission will be amplified as it propagates through the fiber, especially when the pump laser power is increasing. As amplified spontaneous emission (ASE) covers a wide wavelength range 1520-1570nm, we can use it as a broadband light source.

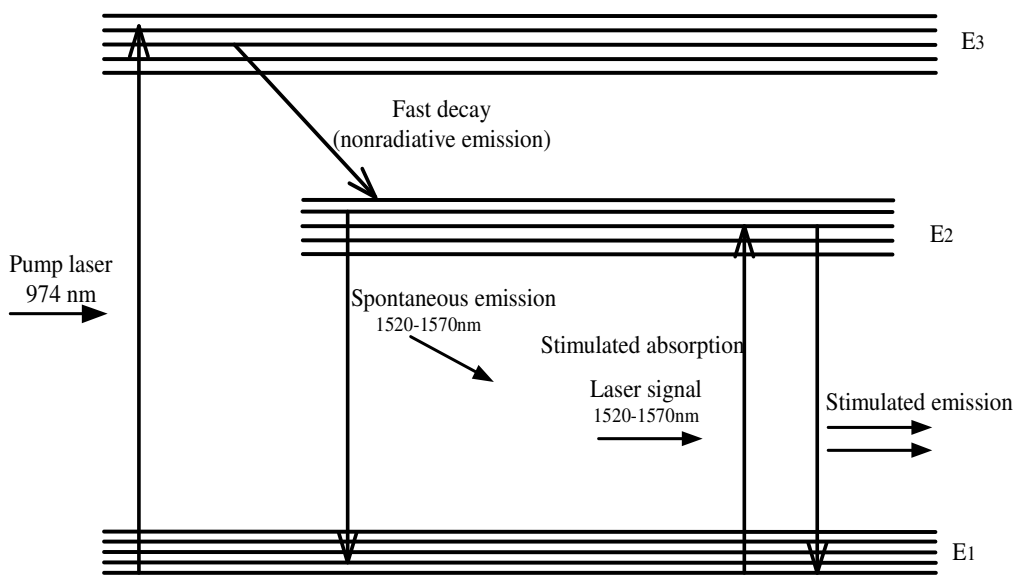

Figure 1 Simplified Energy levels of $\mathrm{Er}^{3+}$ ions in Erbium-doped fiber

If a laser signal with a wavelength between 1520 and $1570 \mathrm{~nm}$, and a 974 pump laser are fed into an erbiumdoped fiber simultaneously as shown in Figure 1, there are three possible outcomes for the signal photon: i) stimulated absorption: signal photon excites an erbium ion from the state $E_{1}$ to a higher level $E_{2}$ and become annihilated in the process; ii) stimulated emission: signal photon stimulates an erbium ion at state $E_{2}$ to decay to $E_{1}$, producing another identical photon. Thus the signal is amplified; iii) signal photon can propagate unaffected through the fiber. In the mean while, spontaneous emission always occurs between level $\mathrm{E}_{2}$ and level $\mathrm{E}_{1}$. When pump laser power is high enough that the population inversion is achieved between the energy level $\mathrm{E}_{2}$ and $\mathrm{E}_{1}$ of erbium-doped fiber, the input laser signal passing through the fiber is then be amplified. Thus we can use erbium-doped fiber and pump laser to construct an optical amplifier, which called erbium-doped fiber amplifier (EDFA). The spontaneous emission also could be amplified by pump laser. So ASE is always present in EDFA, and it is the main source of noise in these amplifiers.

The laser is simply the optical amplifier with positive feedback. If the output of EDFA is fed back to its input to build a fiber loop, when pump laser power is added into the fiber loop, the EDFA is transformed to a fiber laser, which is called erbium-doped fiber laser (EDFL). The laser wavelength varies with the cavity loss. Therefore, the laser wavelength can be tuned by adjusting the cavity loss.

\section{Experiment Set Up}

The schematic diagrams of ASE broadband light source, EDFA, and EDFL experiment setups are shown in Figure 2, Figure 3, and Figure 4, respectively. It is obvious that Figure 2 and Figure 4 are parts of the Figure 3.

In the Figure 3, the eight distributed feed back (DFB) laser sources with eight different wavelengths individually from $\lambda_{1}$ to $\lambda_{8}$ are fed into an $8 \times 8$ tree coupler. The eight wavelengths are:

$$
\begin{array}{llll}
\lambda_{1}=1530.33 \mathrm{~nm}, & \lambda_{2}=1535.04 \mathrm{~nm} & \lambda_{3}=1540.56 \mathrm{~nm} & \lambda_{4}=1545.32 \mathrm{~nm} \\
\lambda_{5}=1550.12 \mathrm{~nm} & \lambda_{6}=1554.96 \mathrm{~nm} & \lambda_{7}=1559.79 \mathrm{~nm} & \lambda_{8}=1564.68 \mathrm{~nm}
\end{array}
$$

The eight outputs from the tree coupler can accommodate eight lab stations. Each output from tree coupler contains eight wavelengths from $\lambda_{1}$ to $\lambda_{8}$ but with a theoretical power loss of $9 \mathrm{~dB}$. One of the outputs from the tree coupler is connected to the input of a Variable Optical Attenuator (VOA). The output from the VOA is considered as the Input Signal of EDFA, which contains the wavelengths $\lambda_{1}$ to $\lambda_{8}$ and the power can be adjusted by different VOA settings. 
The EDFA consists of a WDM component, two optical isolators, an erbium doped fiber strand with 7 meter in length, a pump laser, and a laser diode driver. All are attached on a plexiglass board. The Input Signal transmits through the Optical Isolator 1 to reach the 980/1550 WDM. The Pump Laser (974nm) that mounted on the Laser Diode Mount and driven by the Laser Current Source is fusion spliced to the 980/1550 WDM. The Pump Laser Power versus Drive Current relations should be measured before pump laser output fiber fusion spliced to 980/1550 WDM.

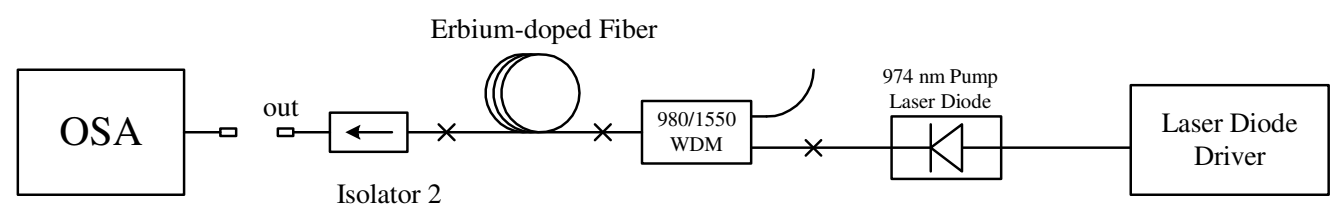

Figure 2 Schematic diagram of ASE broadband light source experiment set up (fusion fiber splices: $x$; fiber connectors: ${ }^{\square}$ )

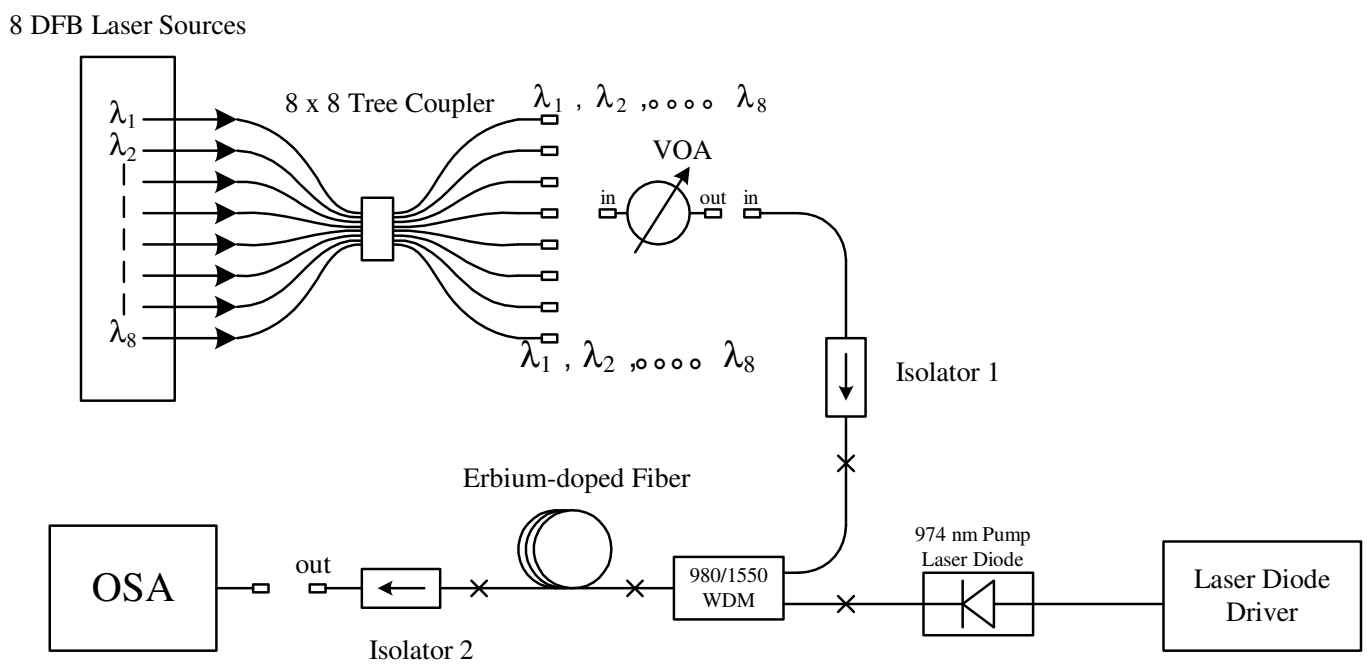

Figure 3 Schematic diagram of multi-wavelength EDFA experiment set up

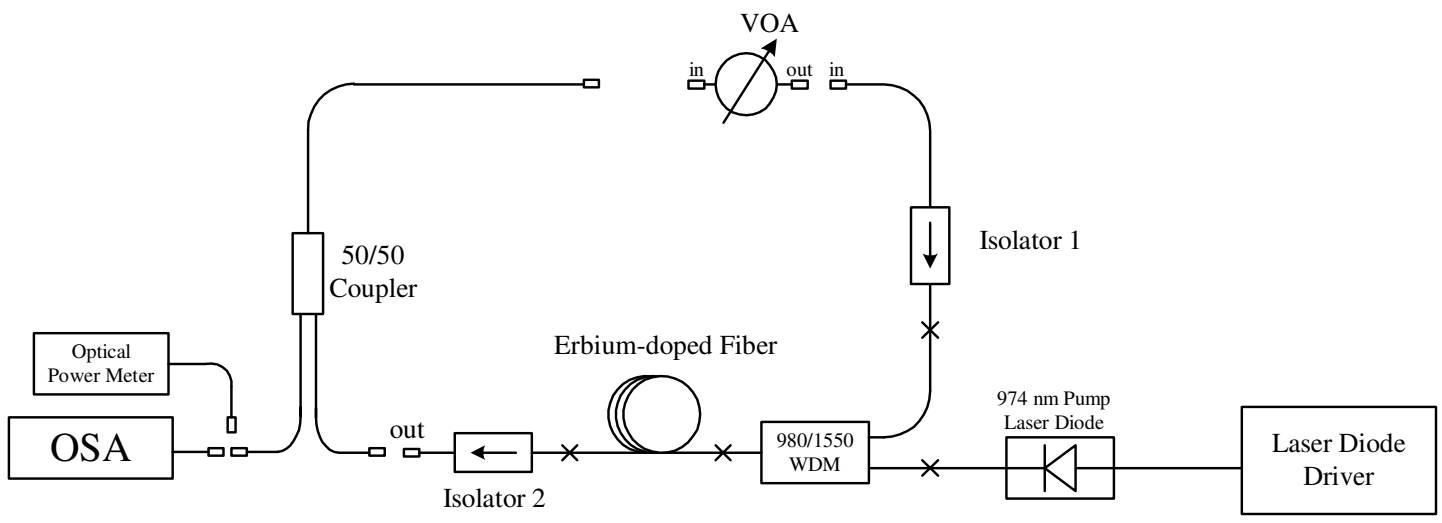

Figure 4 Schematic diagram of EDFL experiment set up 
The Input Signal and Pump Laser are inputted into the 980/1550 WDM separately and come out through the common fiber, and then spliced to an Erbium-doped fiber (7 meter in length, Corning PureMode 1550C Photonic Fiber). The amplified signal passes along another Optical Isolator 2 and is considered as the Output Signal of the EDFA which can be studied by the Optical Spectrum Analyzer (OSA).

\section{Experiment Results and Discussions}

\subsection{Construct an amplified spontaneous emission (ASE) broadband light source and measure its emission spectrum}

An ASE broadband light source is built by using a 974nm laser diode pumping an erbium-doped fiber as shown in Figure 2. Connect the output of the erbium-doped fiber to the Optical Spectrum Analyzer (OSA), gradually increase pump laser diode power. The optical spectra of the amplified spontaneous emission (ASE) at different pump powers are shown in Figure 5. The figure shows that ASE power spectrum covers a broadband wavelength range from 1520 to $1570 \mathrm{~nm}$ under the $974 \mathrm{~nm}$ laser diode pumping. The total ASE power increases with the pump power.

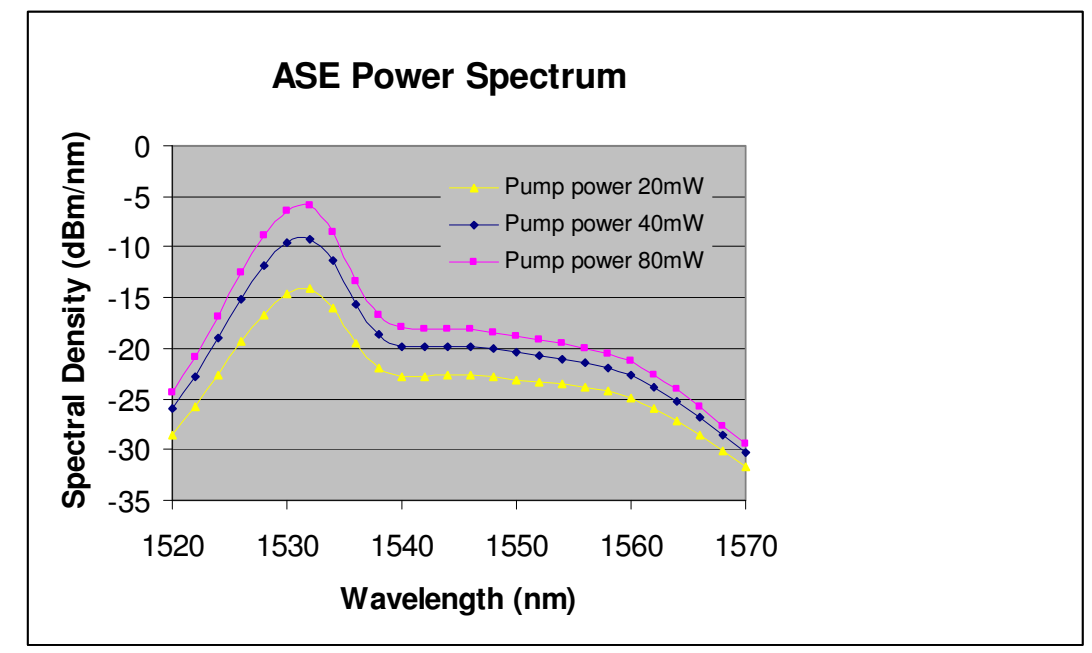

Figure 5 Amplified spontaneous emission (ASE) spectrum of erbium-doped fiber pumped by a $974 \mathrm{~nm}$ laser diode

\subsection{Modify the ASE source into a broadband multi-wavelength erbium doped fiber amplifier (EDFA)}

In this experiment, as shown in Figure 3, connect the output of VOA to the OSA by a patchcord to measure the input signal spectrum, and then connect the output of VOA to the input of isolator 1, connect the output of the isolator 2 to OSA to measure the output signal spectrum. The input signal and output signal spectrum of EDFA at VOA setting $20 \mathrm{~dB}$ and pump power $80 \mathrm{~mW}$ are shown in Figure 6 . The figure highlights the wavelength dependence of the gain and clearly shows how the ASE is added to the output spectrum as noise. The gain profile and noise figure (NF) performances of EDFA under various input and pump parameters are going to be further studied. 

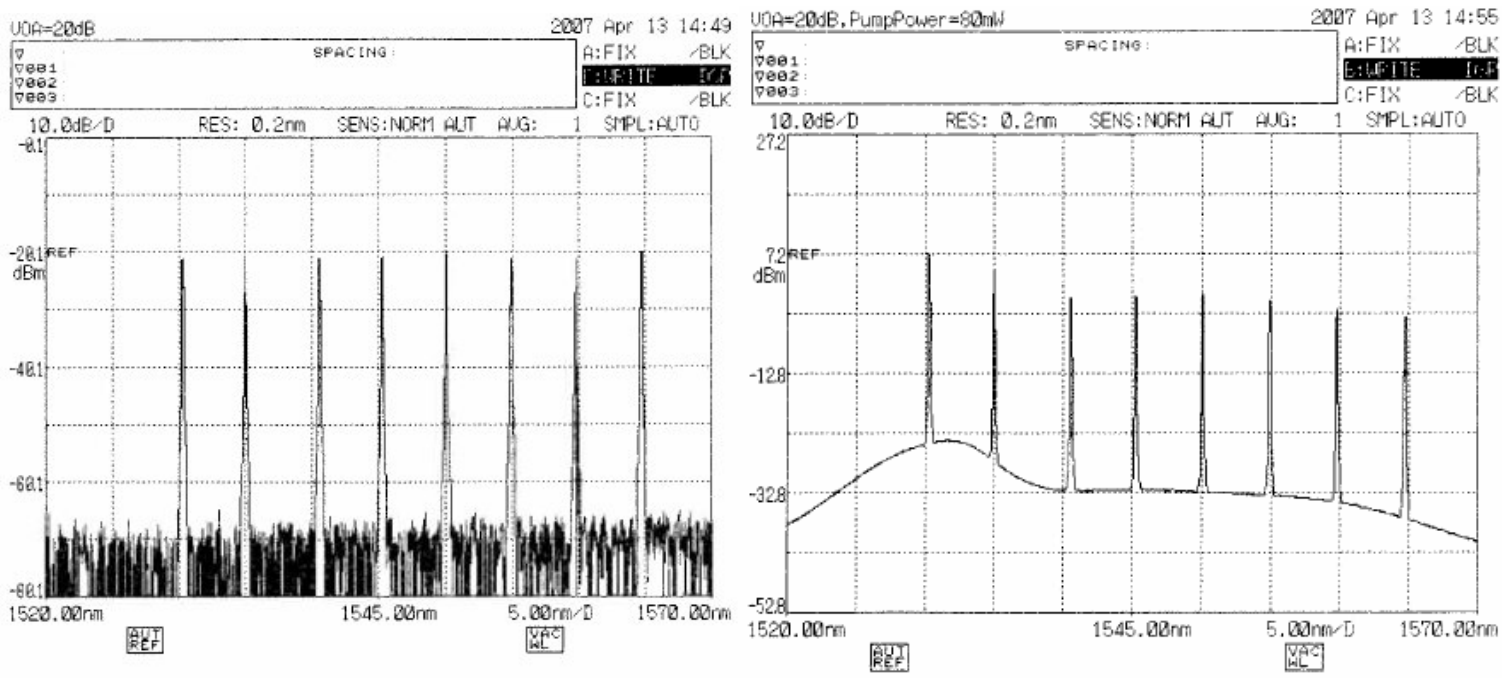

Figure 6 Input (left) and output (right) spectrum of multi-wavelength EDFA @ VOA setting 20dB and pump power $80 \mathrm{~mW}$

\subsubsection{EDFA gain profile versus input signal at fixed pump power}

The peak powers of eight different input wavelengths from $\lambda_{1}$ to $\lambda_{8}$ at different VOA settings: $0,5 \mathrm{~dB}$, and 10dB are measured firstly. Then set the pump power to $80 \mathrm{~mW}$ and measure the output signal powers of each channel at different VOA settings: $0 \mathrm{~dB}, 5 \mathrm{~dB}$, and $10 \mathrm{~dB}$ respectively. The gain profile of EDFA at different input signal is shown in Figure 7.

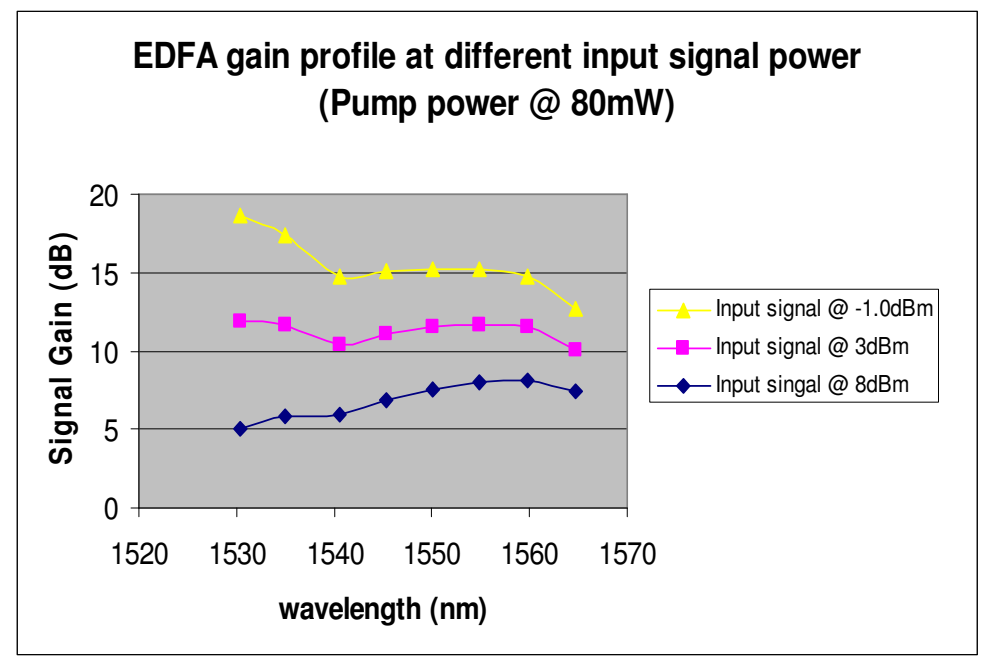

Figure 7 EDFA gain profile

Figure 7 displays the erbium doped fiber has a wide gain spectrum from 1530 t0 $1565 \mathrm{~nm}$. The wide gain spectrum of EDFA has made the use of optical amplifier particularly attractive for DWDM lightwave systems as all channels can be amplified. Figure 7 also displays that the gain profile is wavelength dependent and input signal power dependent. So the design of EDFA with flat gain over the wide wavelength range is crucial for practically use of EDFA.

4.2.2 EDFA gain versus input signal power at fixed pump power for specific channel wavelength $1550.12 \mathrm{~nm}$ 
From Figure 7 we find that for specific input signal wavelength, the signal gain decreases when the input signal power increases. Next, the EDFA gain saturation properties will be studied. In this configuration the output of VOA is connected to the optical spectrum analyzer (OSA) by a fiber patchcord. The input signal peak power at different VOA settings is measured from $40 \mathrm{~dB}$ to $0 \mathrm{~dB}$ in steps of $-5 \mathrm{~dB}$ for channel 5 at wavelength $\lambda_{5}=1550.12 \mathrm{~nm}$. Then the patchcord between VOA and OSA is disconnected. The input side of optical isolator 1 is connected to VOA output and the signal is fed to the EDFA. The output side of optical isolator 2 is connected to OSA. Set the pump power to $100 \mathrm{~mW}$. The output signal powers of wavelength $1550.12 \mathrm{~nm}$ at different VOA settings from $40 \mathrm{~dB}$ to $0 \mathrm{~dB}$ in steps of $-5 \mathrm{~dB}$ are measured.

The EDFA gain versus signal power at fixed $100 \mathrm{~mW}$ pump, and output power versus input power figures are shown in Figure 8, and Figure 9 respectively.

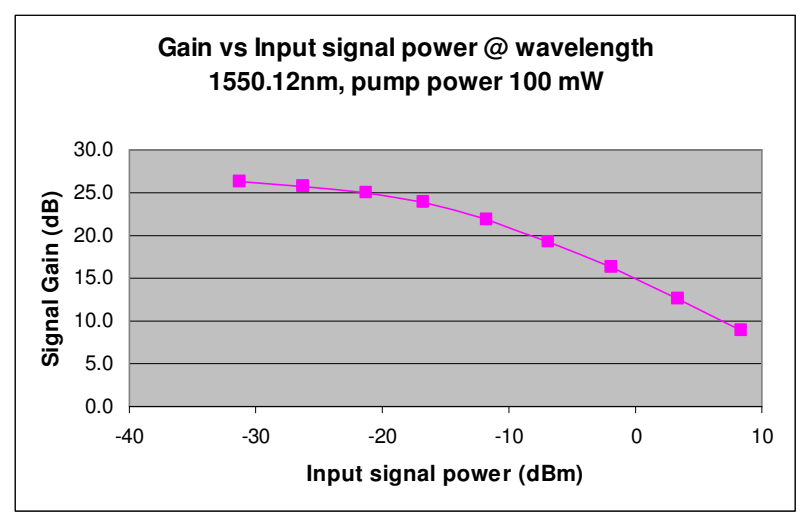

Figure 8 EDFA gain versus input signal

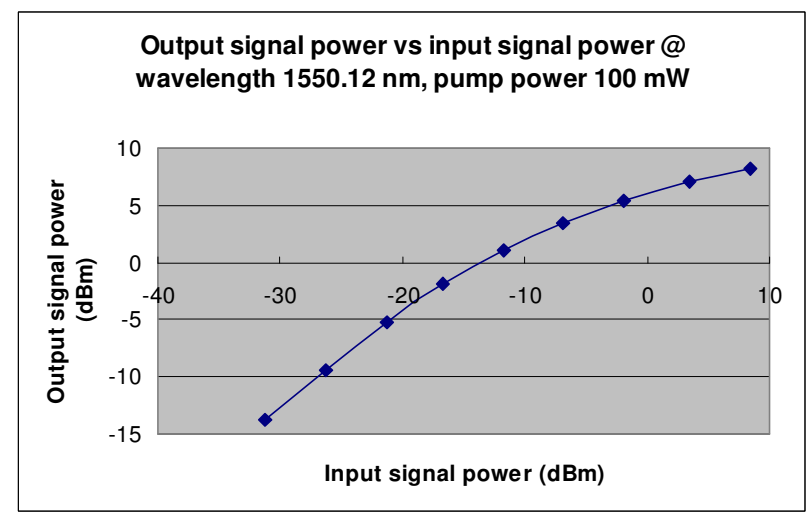

Figure 9 EDFA output power versus input signal power

The gain saturation of EDFA is obviously seen from Figure 8 and Figure 9. Under certain of pump power, the small signal gain keeps constant in a range of input signal power, and hence the output signal power increase linearly with the input signal power. However, the signal gain decreases when signal power further increases, and the output signal power trends to saturate accordingly. This gain saturation happens when all the pump power is consumed. The increase of pump power may increase the small signal gain.

\subsubsection{EDFA gain versus pump power at fixed input signal power for channel wavelength $1550.12 \mathrm{~nm}$}

The VOA is first set to $20 \mathrm{~dB}$, the input signal power at wavelength $1550.12 \mathrm{~nm}$ and total eight wavelength input power are measured. Then the pump power is changed to the values of $2 \mathrm{~mW}, 5 \mathrm{~mW}, 7 \mathrm{~mW}, 10 \mathrm{~mW}, 20 \mathrm{~mW}, 40$ $\mathrm{mW}, 60 \mathrm{~mW}, 80 \mathrm{~mW}$ to $100 \mathrm{~mW}$, which the output signal power at wavelength 1550.12 is measured to calculate the corresponding gain.

The EDFA gain versus pump power is shown in Figure 10. When gradually increasing the pump power, the initial gain is negative, which means stimulated absorption is stronger than stimulated emission. However, the gain increases linearly with the pump power. The threshold pump power where output power is equals to input power (i.e. gain $=0$ ) can be determined. When the pump power continuously increases, the gain increases slowly till saturated, which indicates the pump saturation occurs. At low level of pump power, the population inversion is sufficient to provide gain. While at the high level of pump power, the pump light significantly depletes the population of the ground state. 


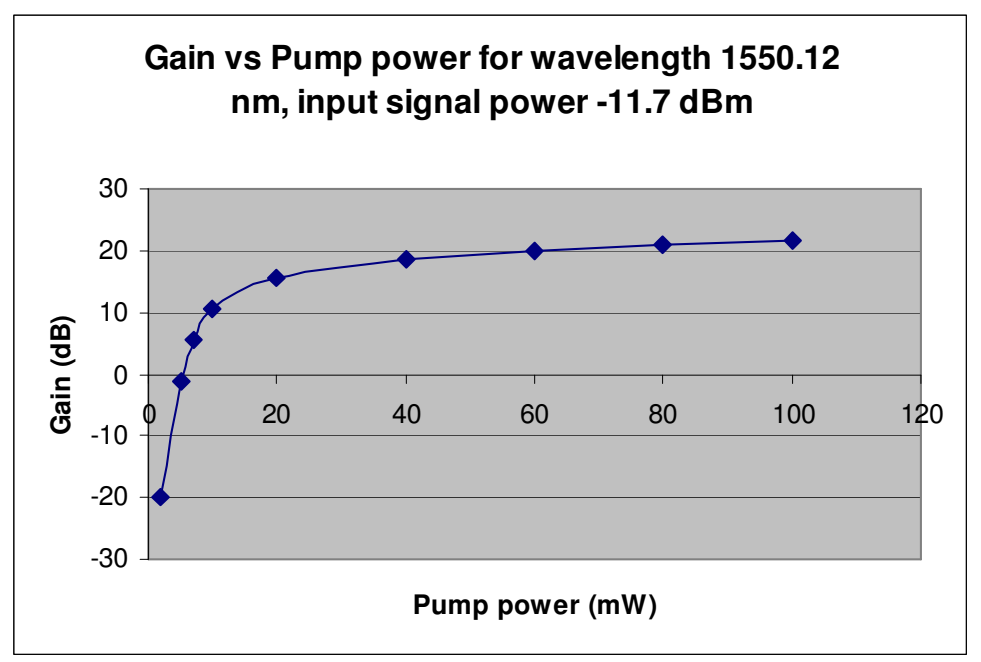

Figure 10 EDFA gain versus pump power

4.2.4 EDFA noise figure (NF) versus pump power at fixed input signal power for specific channel wavelength $1550.12 \mathrm{~nm}$

The noise figure of an amplifier is a measure of the degradation of the signal to noise ratio for a signal passing through the amplifier. The noise figure (NF) is defined as the ratio of the signal to noise at the input of the EDFA to that at the output of the EDFA $\left(\mathrm{NF}=(\mathrm{SNR})_{\text {in }} /(\mathrm{SNR})_{\text {out }}\right)$. It can be expressed as [2]:

$$
N F(d B)=10 \log _{10}\left(\frac{P_{A S E}}{h v \Delta v G}+\frac{1}{G}\right)=10 \log _{10}\left(\frac{\lambda^{3} P_{A S E}}{h c^{2} \Delta \lambda G}+\frac{1}{G}\right) \quad \text { Equation (1) }
$$

where $\lambda$ is the optical wavelength, $\mathrm{h}$ is Plank's constant, $\mathrm{c}$ is the speed of light, $\Delta \lambda$ is the optical bandwidth, $\mathrm{P}_{\mathrm{ASE}}$ is the ASE power (linear units) measured in the bandwidth $\Delta \lambda, \mathrm{G}$ is the gain of optical amplifier (linear units). When use optical spectrum analyzer (OSA) to do measurement, The ASE power $\mathrm{P}_{\mathrm{ASE}}$, optical bandwidth $\Delta \lambda$, peak wavelength $\lambda$, and gain $G$ can be measured by the Analysis Function of the OSA. The NF therefore can be calculated by Equation 1. The EDFA noise figure versus pump power at fixed input signal power for channel 5 is shown in Figure 11.

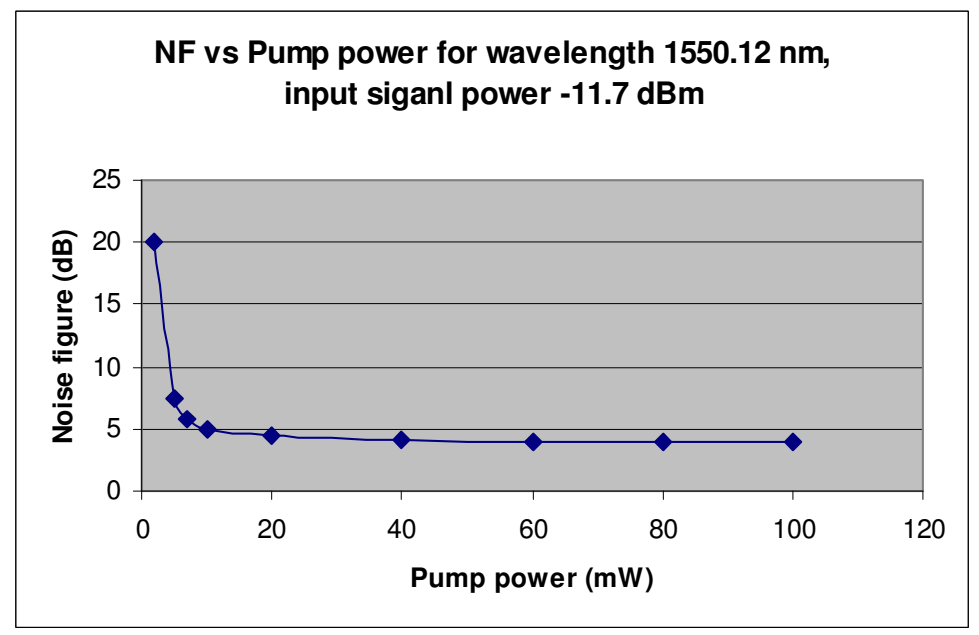

Figure 11 EDFA noise figure (NF) versus pump power 
When the pump power is as low as $2 \mathrm{~mW}$, the NF is as high as $20 \mathrm{~dB}$. A high noise figure indicates that the signal to noise ratio has been impaired by the amplification process. Actually, in this case, the pump power is too lower that the signal gain is negative (see Figure 10), which means stimulated absorption is stronger than stimulated emission between energy level $\mathrm{E}_{2}$ and $\mathrm{E}_{1}$, leading signal be absorbed when passes through EDFA. The NF decreases dramatically as the pump power increases until the pump saturation (see Figure 10) occurs. Although ASE can be used to build up a broadband light source as described in 4.1, the ASE is the main noise in the optical amplifier which can be seen in Figure 6. All amplifiers degrade the signal to noise ratio (SNR) of the amplified signal because of spontaneous emission that adds noise to the signal during the amplification. The degraded NF is one of the major limits for the applications of EDFA in optical communication links and systems.

\subsection{Construct a tunable fiber laser by erbium- doped fiber}

The laser is simply the optical amplifier with positive feedback. As shown in Figure 4, an erbium-doped fiber ring laser can be constructed by connecting the output of EDFA board, i.e. the output side of Isolator 2, to the input of EDFA board, i.e. the input side of Isolator 1, by using $974 \mathrm{~nm}$ laser as a pump laser. In order to adjust the loss of the fiber ring laser, a VOA is inserted between Isolator 1 and Isolator 2. A 50/50 coupler is connected between the output of EDFA board and the input of VOA to measure the fiber laser power and spectrum performance by the optical power meter and OSA, respectively.

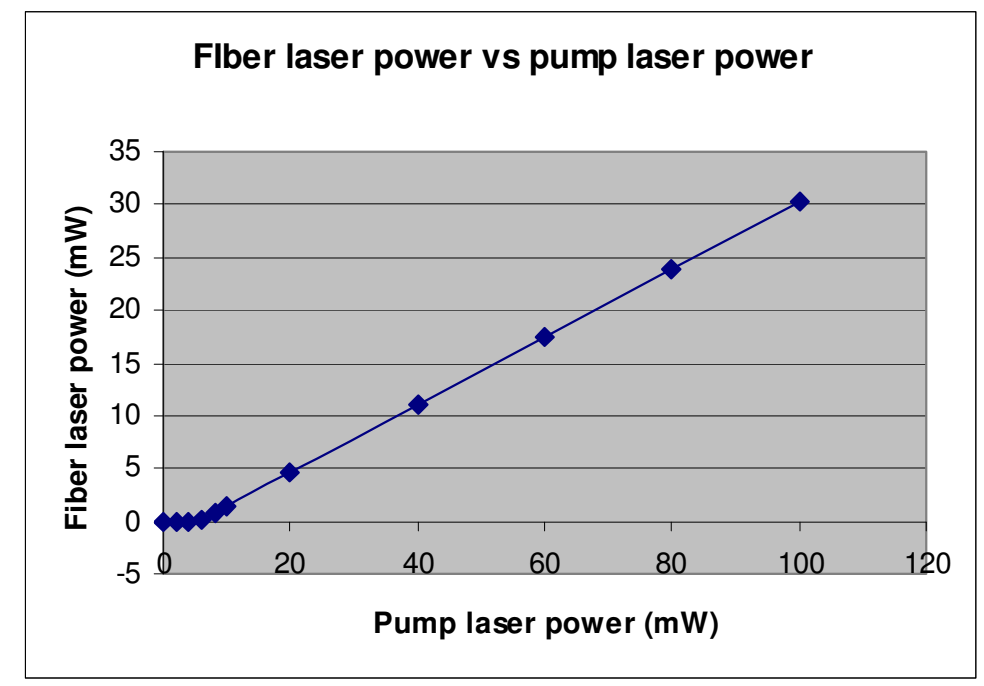

Figure 12 Erbium fiber laser power versus pump power

The fiber laser power versus pump power is shown in Figure12. The threshold pump power can be determined from Figure 12. The fiber laser spectrum can be observed by OSA. Figure 13 shows the laser spectra at which the VOA settings are $9 \mathrm{~dB}$ and $1 \mathrm{~dB}$. The laser peak wavelengths are $1532.92 \mathrm{~nm}$ and $1559.60 \mathrm{~nm}$ respectively. The linewidth of the fiber is as narrow as $0.2-0.5 \mathrm{~nm}$. This implies that the laser wavelength could be tuned by carefully adjust the cavity loss. 

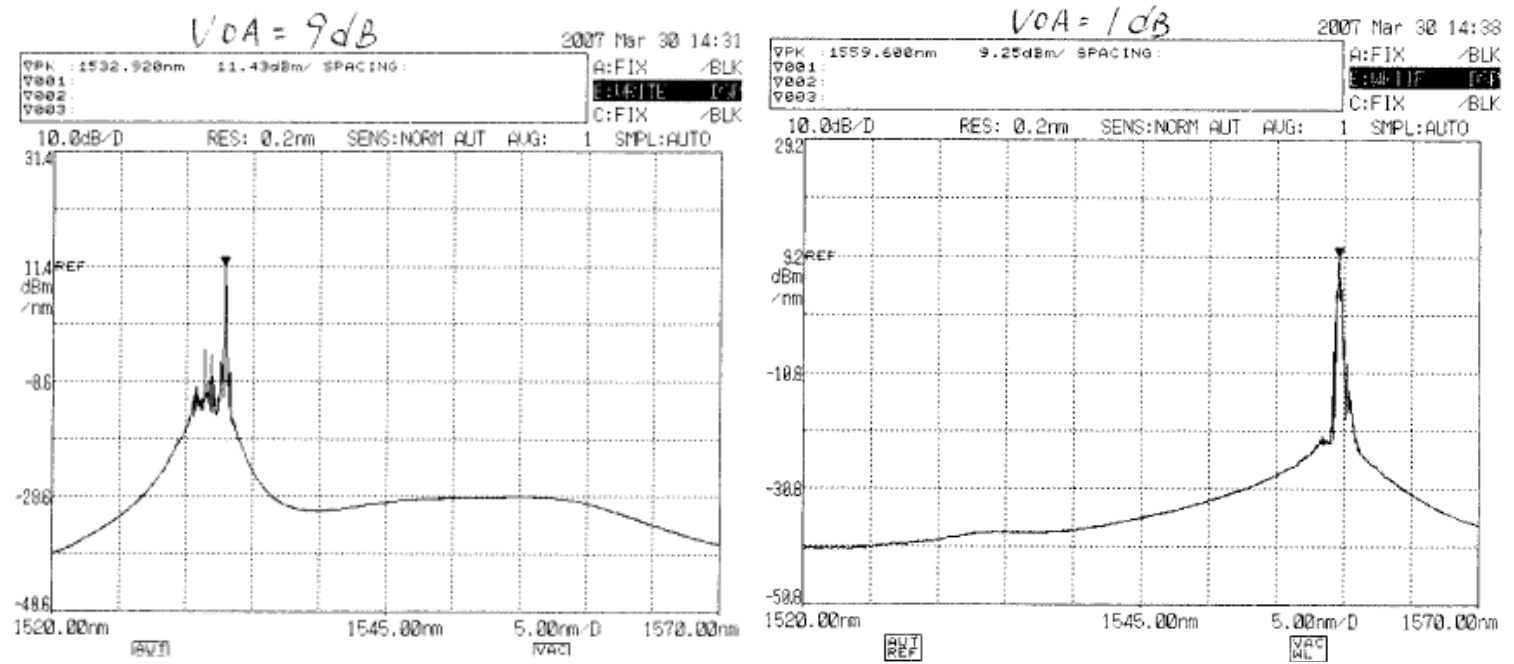

Figure 13 Erbium-doped fiber ring laser spectrum at different fiber loop loss (left: $\mathrm{VOA}=9 \mathrm{~dB}$, right: $\mathrm{VOA}=1 \mathrm{~dB}$ )

\section{Experiment cost}

In order to give our students the exposure to state-of-the-art equipment and yet make them affordable, we have been purchasing refurbished equipment and components and assemble them into a complete system. The devices and components used in this experiment include: ILX FOM-7900B mainframe with eight DFB laser modules in C band, Anritsu MN9605 VOA, ILX LDX3525 laser current source, ILX laser diode mount, Bookham $974 \mathrm{~nm}$ pump laser, Ando 6317 OSA, Newport 1815-C power meter and detector, Corning PureMode 1550C Photonic erbium- doped fiber, isolators, tree coupler, 980/1550 WDM, and 50/50 coupler. The cost of each EDFA board which includes VOA, isolators, WDM, pump laser, erbium-doped fiber, and a coupler is around USD1300. The DFBs cost USD8000, while with an $8 \times 8$ tree coupler (cost USD500), it can accommodate eight lab stations to meet the demand of large scale course. Other equipment such as OSA (cost USD3500) and laser current source (cost USD1300) are very general and powerful equipment and also be used in other experiments in our lab, such as characterization of laser diode and LED, optical absorption and photoluminescence in semiconductors. With these advanced equipments, we can build new experiments conveniently and the cost distributed to each lab will be down.

\section{Conclusions}

The basic theories of erbium doped fiber amplifier and laser are very challenging to undergraduate students. Spontaneous emission, stimulated emission, and stimulated absorption are basic concepts of laser and optical amplifier. In this experiment, we use erbium-doped fiber as gain medium to build three devices: a broadband light source, a multi-wavelength optical amplifier, and a fiber laser.

In the first part of the experiment, students will construct a broadband source through an erbium-doped fiber pumped by a laser diode and measure its emission spectrum. The broadband light source is based on the amplified spontaneous emission and it is a typical example of incoherent light source. In the second part of the experiment, students will build a multi-wavelength erbium-doped fiber amplifier (EDFA) by simply adding the multi-wavelength laser sources to the first part of the experiment setup. The optical amplifier is based on stimulated emission. The laser signals within a wide range $1520-1570 \mathrm{~nm}$ can be amplified without change their coherence properties when pass through an EDFA. To further understand the performance of EDFA, the gain spectrum and noise figure features of EDFA respect to input and pump parameters also be investigated. In the third part of the experiment, students will construct an erbium-doped fiber laser (EDFL) just by connecting the output of the EDFA to the input of EDFA. The laser wavelength is easily be tuned by adjust the laser loop loss. The fiber laser is based on the stimulated emission too with positive feedback. The switches between these three devices are very easy and convenient. From this series of experiments, students will have deep understandings the difference between laser and incoherent light source, the difference of laser and optical amplifier. The theoretical analysis of gain spectrum, gain saturation, noise figure of EDFA as functions of input and pump properties are challenges to undergraduate students. The experimental results from this experiment are very straight forward and illustrative. 


\section{References}

[1] E. Desurvire and J.R. Simpson, "Amplification of Spontaneous Emission in Erbium-Doped Single-Mode Fibers", J. Lightwave Tech., Vol.7, No.5, 835, 1989 .

[2] P.C. Becker, N.A. Olsson, and J.R. Simpson, "Erbium-Doped Fiber Amplifiers", Academic Press, 1999.

[3] G.P Agrwwal, "Fiber-Optic Communication Systems", Wiley Interscience, 3rd ed., 2002.

[4] M. Melo, O. Frazao, A.L.J. Teixeira, L.A. Gomes, J.R. Ferreira D. Rocha, H.M. Salgado, "Tunable L-band erbium-doped fiber ring laser by means of induced cavity loss using a fiber taper", Applied Physics B, Vol.77, 139, 2003.

[5] W. Johnstone, B. Culshaw, D. Walsh, D. Moodie, and I. Mauchline, "Student Laboratory Experiments on Erbium Doped Fiber Amplifiers and Lasers", SPIE Proceedings, $6^{\text {th }}$ International Conference on Education on Training of Optics and Photonics, Vol. 3831, $259,2000$.

[6] R. J. Mears, S. R. Baker, "Erbium Fiber Amplifiers and Lasers", Optical and Quantum Electronics, Vol.24, 517, 1992. 